\title{
Sonography of Affected and Unaffected Shoulders in Hemiplegic Patients: Analysis of the Relationship Between Sonographic Imaging Data and Clinical Variables
}

\author{
Hyong Keun Cho, M.D., Hyoung Seop Kim, M.D., Seung Ho Joo, M.D. ${ }^{1}$ \\ Department of Physical Medicine and Rehabilitation, ${ }^{1}$ Department of Diagnostic Radiology, \\ National Health Insurance Corporation Ilsan Hospital, Goyang 410-719, Korea
}

\begin{abstract}
Objective To explore the relationship between a number of clinically relevant variables and sonographic imaging data in respect to the level of impairment experienced in the affected and unaffected shoulders of hemiplegic stroke patients.

Method Fifty-one hemiplegic stroke patients (32 males, 19 females; 29 right-sided hemiplegics, 22 left-sided hemiplegics) participated in this study. A musculoskeletal radiologist conducted a sonographic exam on both the affected and unaffected shoulders of all patients and two physicians classified the severity of the injury on a six-point rating scale. Clinical variables including age, sex, duration of injury, spasticity and muscle power of the hemiplegic side, and level of functional activity of the shoulder were assessed.

Results The sonographic rating scores of hemiplegic shoulders were positively correlated with age $(p<0.01)$ and negatively correlated with level of muscle spasticity $(\mathrm{p}<0.05)$. The sonographic rating scores of unaffected shoulders were positively correlated with duration of injury $(\mathrm{p}<0.01)$. Affected shoulders received sonographic rating scores that reflected significantly more impairment than those of unaffected shoulders $(\mathrm{p}<0.001)$, and premorbid handedness did not affect the relationship between impairment rating and shoulder injury status.

Conclusion Hemiplegic stroke influences not only affected shoulders, but also unaffected sides. Proper management of spasticity, enhancement of motor recovery, and avoidance of unaffected shoulder overuse should be considered to prevent shoulder problems following strokes which result in hemiplegia.
\end{abstract}

Key Words Ultrasonography, Hemiplegia, Shoulder

Received April 3, 2012; Accepted June 23, 2012

Corresponding author: Hyoung Seop Kim

Department of Physical Medicine and Rehabilitation, National Health Insurance Ilsan Hospital, 100, Ilsan-ro, ilsan-donggu, Goyang 410-719, Korea Tel: +82-31-900-0137, Fax: +82-31-900-0343, E-mail: rekhs@nhimc.or.kr

(c) This is an open-access article distributed under the terms of the Creative Commons Attribution Non-Commercial License (http://creativecommons. org/licenses/by-nc/3.0) which permits unrestricted noncommercial use, distribution, and reproduction in any medium, provided the original work is properly cited.

Copyright $\odot 2012$ by Korean Academy of Rehabilitation Medicine 


\section{INTRODUCTION}

The diagnostic modalities used in the evaluation of shoulder disabilities include X-ray, shoulder-joint arthrography, ultrasonography, computerized tomography (CT), magnetic resonance imaging (MRI), and magnetic resonance (MR) arthrography. ${ }^{1}$

Radiological methods are the most appropriate tools in the evaluation of shoulder pain, particularly when examining bony structures and joint subluxation is suspected. However, these methods cannot be used to examine soft tissue lesions. Shoulder joint arthroscopy, an invasive method, is appropriate for diagnosis of rotator cuff tears. CT scans depict detailed images of bone and, when combined with arthrography, show intra-articular pathology. ${ }^{2}$ The primary, noninvasive methods used to diagnose rotator cuff pathology are sonography and MRI. ${ }^{3} \mathrm{MRI}$ is superior to other diagnostic methods in diagnosing soft tissue lesions, as well as bone marrow lesions. ${ }^{2,4}$ However, MRI is relatively expensive, time-consuming, and inappropriate for the examination of both shoulders at once, which is particularly important when evaluating hemiplegic stroke patients due to their limited range of motion and their need for comfortable positioning in the scanner. ${ }^{3}$

Ultrasonography is a useful diagnostic tool for the assessment of rotator cuff and non-rotator cuff disorders of the shoulder. ${ }^{5,6}$ Ultrasonography is a noninvasive and inexpensive imaging modality that does not expose patients to radiation, and this method allows for the evaluation of structural status of the tissue, as well as the examination of dynamic movement of the affected area. Thus, ultrasonography is an imaging tool which allows for both anatomical and functional assessments of hemiplegic patients. ${ }^{3,7}$

Shoulder pain is a common complication following hemiplegic stroke. This pain may interfere with functional improvement, the patient's quality of life, and it may impede the process of rehabilitation. ${ }^{8}$ Many factors are thought to contribute to hemiplegic shoulder pain. The clinical management of hemiplegic shoulder pain usually involves the administration of oral analgesics, intra-articular injections, and therapeutic exercises. ${ }^{3,9}$ Further understanding of rotator cuff tendon impairment in stroke patients may help clinicians in assigning more complete therapeutic plans to hemiplegic patients. ${ }^{3}$
Following a stroke, immobility and weakness often force patients to compensate for affected body parts by using the unaffected parts in their daily activities. ${ }^{10}$ In this study, we hypothesized that stroke resulting in hemiplegia would result in damage to not only tendons in affected shoulders but also to those on unaffected sides, due to compensatory overuse. Patients may not recognize unaffected shoulder pain due to the greater impact of the hemiplegically-affected shoulder problems. Additionally, these patients may not be able to describe their pain due to aphasia or other forms of cognitive dysfunction which commonly follow stroke. In this study, we performed ultrasonography to observe structural and functional changes in the tendons of both the affected and unaffected shoulders of hemiplegic stroke patients.

The purposes of this study were to examine sonographic imaging ratings of both affected and unaffected shoulders, as well as to explore the relationships between sonographic imaging data and a number of clinical variables in hemiplegic stroke patients.

\section{MATERIALS AND METHODS}

\section{Patients and clinical assessment}

Hemiplegic patients who were admitted to the inpatient rehabilitation department from March 2010 to December 2010 were included in this study. All patients who either refracted from pain on range of motion check of the shoulders or verbally complained of shoulder pain with limitation of range motion of either or both shoulders (less than 180 degrees of shoulder flexion or extension, less than 90 degrees of shoulder external or internal rotation) were eligible for this study. Patients were excluded if they had a history of previous surgical management or a limited range of motion so far as to hinder ultrasonographic evaluation.

Fifty-one hemiplegic stroke patients (32 males and 19 females; mean age, 60 years; age range, 23-81 years) were enrolled. Twenty two patients had left-sided hemiplegia, and 29 had right-sided hemiplegia. Twenty six participants had suffered cerebral infarction, and 25 cerebral hemorrhage.

All clinical data were obtained from initial admission assessment by consensus of senior and junior trainees of physical medicine and rehabilitation.

Assessment of clinical variables included age, sex, 

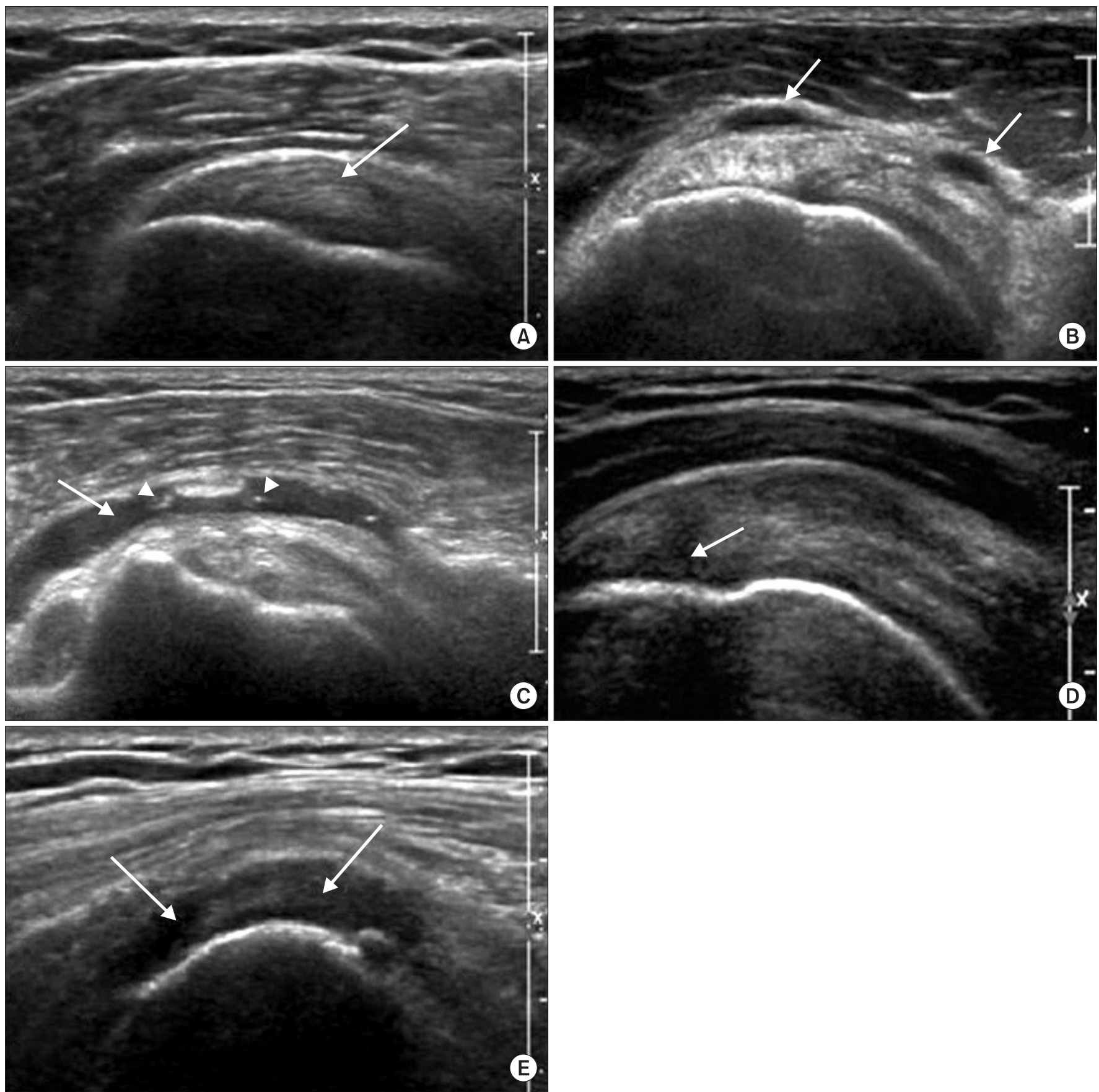

Fig. 1. The scores of sonographic data. (A) Tendinosis of the supraspinatus tendon (grade 1): The long axis view shows slightly decreased echogenicity of the swollen tendon (arrow). (B) Mild bursitis of the subacromail-subdeltoid bursa (SASD) (grade 2): The long axis view shows small amounts of fluid collection (arrows) in the subacromail-subdeltoid bursa. (C) Severe bursitis of SASD bursa (grade 3): The long axis view shows a large amount of fluid collection (arrow) with internal echogenic spots (arrow heads) in the SASD bursa. (D) Articular side partial tear of the supraspinatus tendon (grade 4): The long axis view shows a focal hypoechogenic area (arrow) of the articular side of the supraspinatus tendon. (E) Complete rupture of the supraspinatus and infraspinatus tendons (grade 5): The short axis view shows absence of tendon (arrows) at the greater tubercle of the humeral head. 
handedness, duration of injury, range of motion in the shoulder joints, level of spasticity in the hemiplegic upper extremities, history of use of antispastic agents, level of functional activity of the shoulders, and the nature of the stroke experienced. The level of spasticity in the affected upper extremities was measured using a modified Ashworth scale. Muscle power of the shoulder girdle was examined in each direction, while performing the motions of flexion, extension, adduction and abduction, using the Medical Research Council (MRC) scoring system; these muscle power scores were then summed to give a composite muscle power score. Levels of functional activity were classified as follows: grade 0 , bedridden state; grade 1 , has the ability to roll to one's side; grade 2 , sits alone; grade 3 , stands independently; grade 4 , walks with assistance; grade 5 , walks without assistance.

This study was approved by our Institutional Review Board. All patients and their care givers proved written informed consent.

\section{Ultrasonography and imaging analysis}

Each ultrasonographic examination was performed by a radiologist with three years of experience in musculoskeletal sonography who was unaware of the clinical details of the patients while interpretations were performed to reflect the consensus of one radiologist and one physiatrist. Sonography was performed using a 12-17 MHz high-resolution electronic linear-array transducer (iU22 ultrasound system, Philips Healthcare) with optimized settings and automatic variable frequency adjustment depending on the focal depth. The ultrasonographic evaluation included the subacromial-subdeltoid bursa and the rotator cuffs, which are routinely imaged in both short and long axis planes.

All patients underwent shoulder sonography in a seated position in a wheelchair. Both shoulders were examined in all patients using the scanning techniques described above. ${ }^{3,11,12}$ In patients with limited motion in the hemiplegic shoulder joint, affected shoulder pain, or cognitive dysfunction, the shoulder girdle was examined placed in position similar to that used in the dynamic examination; however, passive movement was induced for these patients with the help of an assistant. None of the patients had shoulder joint limitations that did not allow for a proper six-point rating scale evaluation.

To better define the relationships between ultraso- nographic findings and the clinical variables assessed, ultrasonographic imaging data were reclassified into six grades based on the presence of a rotator cuff tear and fluid collection in the subacromial-subdeltoid bursa or the wall of the shoulder. This rating system has received support from previously published research. ${ }^{3,13-15}$ On this rating scale, a grade of 0 represents no abnormal finding around the rotator cuff; a grade of 1 represents tendinosis of the intratendinous hypoechoic areas with a loss of fibrillar echoes, reflecting a disorganized structure of the collagen bundles of the supraspinatus tendon ${ }^{8,14}$ (Fig. 1-A); a grade of 2 represents a subacromial-subdeltoid bursa thickness of $1.5 \mathrm{~mm}$ to $2.0 \mathrm{~mm}$ (Fig. 1-B); a grade of 3 reflects a subacromial-subdeltoid bursa thickness over $2 \mathrm{~mm}^{15}$ (Fig. 1-C); a grade of 4 indicates a partial or fullthickness tear of the supraspinatus tendon (Fig. 1-D); and a grade of 5 suggests multiple ruptures of the tendons of the rotator cuff (Fig. 1-E) ${ }^{3,15}$ If there were more than two abnormal ultrasonographic ratings for any case, the case was classified based on the most severe rating score. ${ }^{3}$

\section{Statistical analysis}

Statistical analysis was conducted using SPSS for Windows, version 14.0 (SPSS Inc., Chicago, USA). To examine the relationship between ultrasonographic rating scores and clinical variables, Spearman's correlation coefficients were calculated. To compare the ultrasonographic rating scores of affected hemiplegic shoulders and unaffected shoulders, Wilcoxon rank-tests were used. To compare ultrasonographic rating scores based on sex, hemiplegic affected side, and the nature of the stroke, a Mann-Whitney U-test was used. Null hypotheses of no difference were rejected if $p$-values were less than 0.05 .

\section{RESULTS}

The general characteristics of the study participants are summarized in Table 1.

The ultrasonographic rating scores of hemiplegic shoulders were positively correlated with age $(0.35, \mathrm{p}<0.01)$ and negatively correlated with level of spasticity in the hemiplegic upper extremities $(-0.326, \mathrm{p}<0.05)$ (Table 2). The ultrasonographic ratings scores of unaffected shoulders were positively correlated with duration of injury $(0.363, \mathrm{p}<0.01)$ (Table 2). However, ultrasonographic ratings of both affected and unaffected shoulders were not 
Table 1. General Patient Characteristics (Total Number=51)

\begin{tabular}{lc}
\hline \multicolumn{1}{c}{ Clinical variables } & $59.5 \pm 13.1$ \\
\hline Age (year; mean \pm standard deviation) & $32: 19$ \\
\hline Sex (male : female) & 35 \\
Hypertension & 14 \\
\hline Diabetes mellitus & $29: 22$ \\
Hemiplegic side (right : left) & $28: 23$ \\
Nature of stroke (infarction : hemorrhage) & 2 \\
Number of patients taking antispasticity agents & $49: 2$ \\
Handedness (right : left) & $5.5 \pm 5.4$ \\
Injury duration (from onset to sonogram; months) & $19.5 \pm 11.0$ \\
Mini-mental status examination score (mean \pm standard deviation) & $1(0-3)$ \\
Grade of upper extremity spasticity (median; range) & $8(0-16)$ \\
Composite muscle power scores of affected shoulder (median; range) & $3(1-4)$ \\
\hline Functional level (median; range) & \\
\hline
\end{tabular}

Table 2. Correlational Analyses for Clinical Variables and Sonographic Rating Scores of Both Shoulders

\begin{tabular}{llc}
\hline & & Spearman's rho coefficient \\
\hline Spasticity & Affected shoulder & $-0.326^{*}$ \\
& Unaffected shoulder & -0.287 \\
Age & Affected shoulder & $0.358^{\dagger}$ \\
\hline Level of functioning & Unaffected shoulder & 0.025 \\
& Affected shoulder & -0.183 \\
Composite muscle power score of shoulder & Unaffected shoulder & -0.186 \\
\hline \multirow{2}{*}{ Injury duration } & Affected shoulder & -0.145 \\
& Unaffected shoulder & -0.186 \\
\hline
\end{tabular}

${ }^{*}$ Correlation was significant at 0.05 level, ${ }^{\dagger}$ Correlation was significant at 0.01 level

Table 3. Comparison of Sonographic Rating Scores of Both Shoulders by Sex, Hemiplegic Affected-side, and the Nature of Stroke

\begin{tabular}{lcc}
\hline \multicolumn{1}{c}{ Sonography of shoulder lesion } & Variables & p-value \\
\hline Sonographic rating of affected shoulder & Male : Female & 0.79 \\
Sonographic rating of affected shoulder & Right : Left & 0.62 \\
Sonographic rating of affected shoulder & Infarction : Hemorrhage & 0.40 \\
Sonographic rating of unaffected shoulder & Right : Left & 0.77 \\
\hline
\end{tabular}

related to level of functioning or to the composite muscle power score for the shoulder girdle.

Hemiplegic shoulders (median grade $=2$ ) were significantly more impaired than unaffected shoulders (median grade $=1 ; \mathrm{p}<0.001$, Wilcoxon rank-test) based on the ultrasonographic ratings scores. However, sex, side of hemi- plegia, and the nature of the stroke did not influence the relationship between the status (affected or unaffected) of the shoulder and ultrasonographic rating score (Table 3; p>0.05, Mann-Whitney U-test). 


\section{DISCUSSION}

The prevalence of hemiplegic shoulder pain following stroke ranges from $20 \%$ to $84 \%$ of patients. Shoulder pain hinders recovery of function in the upper extremities and increases the duration of hospital stays for patients. ${ }^{4,8,16}$ Shoulder pain following stroke has many causes, such as rotator cuff disease, adhesive capsulitis of the shoulder joint, shoulder joint subluxation, spasticity in the upper extremities, changes in pain sensitivity, complex regional pain syndrome, and centralized post-stroke pain. ${ }^{3,17}$ Although numerous studies have suggested possible mechanisms underlying the development of hemiplegic shoulder pain, the pathophysiology of this disability is still poorly understood. ${ }^{3,9}$ It is important for physicians to define the cause and mechanism underlying the development of hemiplegic shoulder pain in order to help patients achieve a positive rehabilitation outcome. ${ }^{9}$ After hemiplegic stroke, abnormal neuromuscular features induce compensatory adaptations, which may influence the structures and functions of unaffected and affected extremities in hemiplegics. In this study, we hypothesized that hemiplegic stroke affects on not only the shoulder affected by the hemiplegia, but also the unaffected shoulder.

In our study, ultrasonographic rating scores of affected shoulders were positively correlated with patient age and negatively correlated with level of spasticity in the hemiplegic upper extremities. These findings suggest that elderly individuals' rotator cuff tendons may be more prone to injury than those of younger individuals, due to progressive degeneration in aged tendons occurring prior to stroke. In comparison, as the unaffected shoulder was not statistically significantly correlated with age, we can assume that the patient group did not have any premorbid shoulder injuries, and that patients are more prone to postmorbid rotator cuff injuries with increasing age due to greater magnitudes of weaknesses caused by stroke. Additionally, it was determined that enhanced muscle tone in the upper extremities following stroke may have a protective role against injury of rotator cuff tendons. During the flaccid stage immediately after a stroke, lack of muscle tone or muscle paralysis often results in impairment of dynamic control and supportive functions of the rotator cuff. This stage is often followed by abnormal scapulohumeral rhythm, which induces subluxation and increased glenohumeral friction in the hemiplegic shoulder. As a result, the stretch force, caused by abnormal scapulohumeral rhythm, to the flaccid shoulder can cause substantial muscle and tendon injury in the rotator cuff. $^{9,18}$

Previous research suggests that increased spasticity in the upper extremities produces abnormal movement patterns in hemiplegic shoulders, one possible cause of hemiplegic shoulder pain. ${ }^{3,9,19}$ Spasticity in the shoulder muscles may cause pain not only by interfering with normal scapulohumeral movement, but also by increasing the likelihood of contractures. Once contractures have developed, stretching movements in the contracted joints could cause a cycle of pain and reflexive protective spasms. ${ }^{20}$ In this study, ultrasonographic rating scores of affected shoulders were negatively correlated with spasticity levels. This finding suggests that as spasticity develops, muscular activity of the supraspinatus tendon may reduce inferior shoulder subluxation, provoking injury in the rotator cuff tendon due to prolonged overstretching by gravity or direct damage during the flaccid stage..$^{8,21}$ These results also suggest that early recovery of muscle tone in hemiplegic shoulders decreases shoulder subluxation. Thus prevention of rotator cuff tendon injury should be of particular concern during the prolonged flaccid stage.

In a study by Lee et al., ${ }^{3}$ the ultrasonographic rating scale was compared with the Brunnstrom stages scale. This study reported that there was no relationship between ultrasonographic ratings and the stage of motor recovery measured by Brunnstrom stages scale. Brunnstrom classified the pattern of motor recovery after brain injury into six stages according to spasticity level. ${ }^{22,23}$ Subjects enrolled in the study by Lee et al. were assigned a Brunnstrom stage of less than 3, suggesting that each participant in the study experienced prominent spasticity. We suspect that the Brunnstrom stage scale for spasticity level has limited utility with respect to ultrasonographic ratings of shoulder functioning in stroke patients. Spasticity level change of Brunnstrom classification is ambiguous and reflects motor functions of not only the upper extremities, but also the lower extremities, which are not linked to shoulder functioning.

In our study, ultrasonographic rating scale scores for unaffected shoulders were positively correlated with duration of injury. Hemiplegics have a tendency to use 
their unaffected shoulders before achieving adequate motor recovery in their affected sides, which results in prolonged overuse of the unaffected shoulder. Moreover, immediately following stroke, the affected side tends to be hypotonic and in a lowered or "slouched" state compared to the relatively hypertonic and elevated state of the unaffected side. The slouching of the affected side leads to an imbalanced hemiplegic body posture, which can induce rotator cuff tendon injury.

Even though 45 patients (95.7\%) enrolled in this study were right-handed, there were no significant differences between ultrasonographic ratings in pre-morbid dominant and non-dominant shoulders. If degenerative changes in the dominant shoulder tendons occurred prior to stroke, then the ultrasonographic rating scores of the dominant unaffected sides might be assigned with ultrasonographic ratings reflecting greater impairment than those of the non-dominant unaffected sides. However, no significant differences in ultrasonographic ratings between pre-morbid dominant and non-dominant shoulders were found.

Sonography is a useful and readily available method for evaluating shoulder pain and disability. This technique offers the option of dynamic examination, in addition to static evaluation, allowing clinicians to focus on the precise area of maximum discomfort. ${ }^{24,25}$ In spite of the strengths of sonography, its utility greatly depends on the skill and experience of the operator. This method is limited in its ability to assess problems in the articular portions of rotator cuffs, the entire glenohumeral joint, and the anterior glenoid labrum. ${ }^{6,26}$

Several limitations of the current study design should be considered. The duration of injury was relatively heterogeneous among the patients of this study. Thus, it will be beneficial to enroll a more homogeneous group of patients in future studies in order to replicate and validate our results. A longitudinal evaluation of ultrasonographic rating scores was lacking in this study; we should investigate the structure and functioning of both the affected and unaffected shoulders of these patients in a followup study. Most stroke events occur in old age and rotator cuff tendons in the elderly also become degenerative. Thus, comparison of rotator cuff tendons between the hemiplegic side and intact side can be influenced by the degenerative state of the rotator cuff tendons before the onset of stroke. Another limitation is the fact that only one, not two, radiologist had performed the studies, and thus it was not possible to confirm interrater and intrarater reliability.

Also, the patients' current levels of cognitive functioning were not taken into consideration when examining the data. A patient with better cognitive function, who does not have aphasia, can complain of his or her symptoms and utilize an intact upper limb more frequently to perform activity of daily living. These conditions affect both shoulder rotator cuff tendons. In future studies, it will be beneficial to include level of cognitive functioning as one of the clinical variables. Finally, our study was a cross-sectional study, and though we were able to predict that the time since onset influenced the unaffected shoulder, we can only assume that it was caused by overuse. Thus a prospective study on determining the cause of rotator cuff injury at the unaffected shoulder is needed.

In conclusion, hemiplegic stroke results in injury to not only the affected shoulder, but also to the shoulder on the unaffected side. Greater spasticity of the affected limb can be regarded as a preventive factor of rotator cuff tendon from injury and degenerative change. It is suggested that proper management of spasticity through minimal antispastic measures for optimal performance and function without resulting in decrease in muscle tone so far as to cause subluxation combined with avoidance of overuse of the unaffected shoulder will be helpful for patients in the prevention of shoulder injuries following hemiplegic stroke.

\section{REFERENCES}

1. Ko MH, Kim JY, Park SH, Kim NG, Seo JH. Comparison of ultrasonographic findings with clinical findings in hemiplegic shoulder. J Korean Acad Rehab Med 2006; 30: 213-218

2. Tallroth K. Shoulder imaging. A review. Ann Chir Gynaecol 1996; 85: 95-103

3. Lee IS, Shin YB, Moon TY, Jeong YJ, Song JW, Kim DH. Sonography of patients with hemiplegic shoulder pain after stroke: correlation with motor recovery stage. AJR Am J Roentgenol 2009; 192: W40-44

4. Hong JY, Jun PS, Son YG, Choi HW, Lee JH, Kang SH, Kim IT. Magnetic resonance arthrographic findings of the painful hemiplegic shoulder. J Korean Acad Rehab 
Med 2008; 32: 657-663

5. Papatheodorou A, Ellinas P, Takis F, Tsanis A, Maris I, Batakis N. Us of the shoulder: rotator cuff and nonrotator cuff disorders. Radiographics 2006; 26: e23

6. Rutten MJ, Jager GJ, Blickman JG. From the rsna refresher courses: US of the rotator cuff: pitfalls, limitations, and artifacts. Radiographics 2006; 26: 589-604

7. Yang CP, Lee CL, Chen TW, Lee S, Weng MC, Huang $\mathrm{MH}$. Ultrasonographic findings in hemiplegic knees of stroke patients. Kaohsiung J Med Sci 2005; 21: 70-77

8. Turner-Stokes L, Jackson D. Shoulder pain after stroke: a review of the evidence base to inform the development of an integrated care pathway. Clin Rehabil 2002; 16: 276-298

9. Lo SF, Chen SY, Lin HC, Jim YF, Meng NH, Kao MJ. Arthrographic and clinical findings in patients with hemiplegic shoulder pain. Arch Phys Med Rehabil 2003; 84: 1786-1791

10. Bonifer N, Anderson KM. Application of constraintinduced movement therapy for an individual with severe chronic upper-extremity hemiplegia. Phys Ther 2003; 83: 384-398

11. Moosikasuwan JB, Miller TT, Burke BJ. Rotator cuff tears: clinical, radiographic, and US findings. Radiographics 2005; 25: 1591-1607

12. Teefey SA, Middleton WD, Yamaguchi K. Shoulder sonography. State of the art. Radiol Clin North Am 1999; 37: 767-785

13. Schweitzer ME, Karasick D. MR imaging of disorders of the Achilles tendon. AJR Am J Roentgenol 2000; 175: 613-625

14. Bianchi S, Martinoli C. Ultrasound of the musculoskeletal system, Heidelberg: Springer, 2007, 248-259

15. Holsbeeck MT, Introcaso JH. Muculoskeletal ultrasound, 2nd ed, Philadephia: Mosby, 2001, 474-487

16. Lim JY, Koh JH, Paik NJ. Intramuscular botulinum toxin-a reduces hemiplegic shoulder pain: a randomized, double-blind, comparative study versus intraarticular triamcinolone acetonide. Stroke 2008; 39: 126-

\section{1}

17. Bender L, McKenna K. Hemiplegic shoulder pain: defining the problem and its management. Disabil Rehabil 2001; 23: 698-705

18. O'Sullivan S, Schmitz T. Physical rehabilitation, 5th ed, Philadelphia: FA Davis, 2007, 753

19. Van Ouwenaller C, Laplace PM, Chantraine A. Painful shoulder in hemiplegia. Arch Phys Med Rehabil 1986; 67: $23-26$

20. Griffin J, Reddin G. Shoulder pain in patients with hemiplegia. A literature review. Phys Ther 1981; 61: 1041-1045

21. Chaco J, Wolf E. Subluxation of the glenohumeral joint in hemiplegia. Am J Phys Med 1971; 50: 139-143

22. Delisa JA, Gans BM. Physical medicine and rehabilitation, 4th ed, Philadelphia: Lippincott Williams \& Wilkins, 2005, 1667

23. Gowland C, Torrewin W, Van Hullenaar S. Therapeutic exercise for stroke patient. In: Basmajian JV, Wolf SL, Editors. Therapeutic exercise, 15th ed, Baltimore: Lippincott Williams \& Wilkins, 1990, 207-230

24. Read JW, Perko M. Shoulder ultrasound: diagnostic accuracy for impingement syndrome, rotator cuff tear, and biceps tendon pathology. J Shoulder Elbow Surg 1998; 7: 264-271

25. O'Connor PJ, Rankine J, Gibbon WW, Richardson A, Winter F, Miller JH. Interobserver variation in sonography of the painful shoulder. J Clin Ultrasound 2005; 33: 53-56

26. Farin PU, Jaroma H. Sonographic findings of rotator cuff calcifications. J Ultrasound Med 1995; 14: 7-14

27. Rizk TE, Christopher RP, Pinals RS, Salazar JE, Higgins C. Arthrographic studies in painful hemiplegic shoulders. Arch Phys Med Rehabil 1984; 65: 254-256

28. Snels IA, Beckerman H, Twisk JW, Dekker JH, Peter De Koning, Koppe PA, Lankhorst GJ, Bouter LM. Effect of triamcinolone acetonide injections on hemiplegic shoulder pain: a randomized clinical trial. Stroke 2000; 31: 2396-2401 